\title{
Tests de Selection des Spermatozoïdes avant Assistance Médicale à la Procréation
}

\author{
C. Mathieu (1) eT H. Lucas (2)
}

1Laboratoire de Biologie de la Reproduction, Hôpital Pellegrin, 33076 Bordeaux ; 2 Service d'Histologie Embryologie, Biologie de la Reproduction, Hôpital Cochin, 75014 Paris

\section{RESUME}

Les techniques de sélection des spermatozoïdes humains sont nombreuses et souvent spécifiques du centre d'andrologie qui les pratique. Nous ne faisons pas ici l'inventaire de toutes ces techniques mais reprenons les principales, le lavage-centrifugation, les méthodes de filtration ("SpermPrep"), la migration ascendante ainsi que les gradients de densité avec des solutions colloïdales («Percoll», «PureSperm» et «Isolate») ou avec de nouvelles molécules iodées non colloïdales («OptiPrep»). Nous tentons de faire une synthèse de la littérature afín de dégager des méthodologies standardisées par technique et nous discutons les avantages et les inconvénients de chacune d'elle. La France étant un pays où les milieux utilisés pour une assistance médicale à la procréation doivent avoir été agréés d'un point de vue microbiologique, certains milieux décrits ici («Percoll») n'ont pas reçu cet agrément et ne doivent pas être utilisés en thérapeutique alors qu'un usage diagnostique est possible.

Certaines anomalies du sperme nécessitent un type de préparation particulière. Nous décrivons les techniques les plus consensuelles pour traiter les spermes avec anticorps anti-spermatozoïdes, avec présence de leucocytes ou suspects d'infection, les spermes hypervisqueux, ou encore le recueil des spermatozoïdes dans l'urine quand il existe une éjaculation rétrograde. Nous décrivons plus en détail une technique de préparation des spermes cryoconservés en s'inspirant des résultats de recherches fondamen- tales sur la congélation-décongélation des gamètes mâles humains.

Enfin, nous proposons une conduite à tenir pour le choix d'une technique d'AMP à proposer à un couple en tenant compte des caractéristiques du sperme, du résultat du test de sélection spermatique ainsi que de la survie des gamètes.

Mots clés : Sperme humain, préparation du sperme, test de migration survie, gradients de densité, Percoll, migration ascendante.

\section{INTRODUCTION}

Le traitement du sperme in vitro en vue d'une Assistance Médicale à la Procréation (AMP) a pour but de sélectionner des spermatozoïdes mobiles à morphologie normale, d'éliminer le plasma séminal, les débris cellulaires, les cellules (telles que les leucocytes) ainsi que les spermatozoïdes morts et dans une certaine mesure d'amorcer la capacitation. Cette sélection se fait naturellement dans les voies génitales féminines, aboutissant à la présence sur le site de la fécondation de spermatozoïdes susceptibles d'être les plus fécondants de l'éjaculat. La préparation du sperme in vitro essaie de reproduire cette action mais les moyens utilisés peuvent avoir des effets secondaires délétères sur les spermatozoïdes et ce d'autant plus que le sperme sera initialement altéré. La méthode de séparation du sperme choisie sera 
donc un compromis entre le rendement de la sélection et un minimum d'effets secondaires. Le résultat obtenu permettra d'orienter vers un type d'AMP : insémination intra utérine, fécondation in vitro conventionnelle ou avec micromanipulation.

\section{CHOIX DES MILIEUX UTILISES EN AMP}

Les techniques mises en œuvre doivent répondre à des critères d'innocuité et de rendement ; si certains réactifs et milieux peuvent être utilisés au cours de tests de sélection à objectif diagnostic, ils ne peuvent en revanche faire l'objet d'un emploi à usage thérapeutique chez l'homme. Les conditions d'utilisation sont précisées par les fabricants et par la Direction Générale de la Santé (DGS) qui diffuse régulièrement les avis du groupe d'experts de sécurité microbiologique pour les milieux utilisés en AMP contenant des dérivés humains ou animaux.

Les techniques et milieux utilisés pour la préparation du sperme sont habituellement évalués sur des critères de rendement et de mobilité des gamètes. Cependant, d'autres fonctions du spermatozoïde peuvent subir les conséquences de ces manipulations en particulier au niveau membranaire. Par exemple, certains milieux utilisés en FIV peuvent exposer les spermatozoïdes à des peroxydations membranaires [32]. De même la présence d'HEPES dans les milieux de préparation du sperme n'est peut être pas souhaitable. En effet, l'HEPES est connu pour diminuer l'incorporation cellulaire de molécules nécessaires à la synthèse glycoprotéique. Le spermatozoïde étant une cellule hautement différenciée et mature, l'incorporation de ces molécules n'est peut être pas un handicap pour le spermatozoïde. Par contre, l'HEPES modifie le potentiel des membranes cellulaires, bloque certains canaux chlore, affecte les systèmes de transport transmembranaires de molécules, interfêre avec le calcium ionisé, favorise la genèse de radicaux libres et peut inhiber l'utilisation de certains acides aminés par la cellule $[6,12,20$, $22,43,47,64,69,79]$. Tous ces résultats expérimentaux ont été obtenus sur d'autres types cellulaires que les spermatozoïdes mais l'usage de l'HEPES pourrait potentiellement perturber la déstabilisation membranaire spermatique, le déclenchement de la réaction acrosomique et donc la fécondance des gamètes mâles. Même si ce composant est utilisé depuis de nombreuses années, une évaluation de ses effets spermatiques s'impose à nous.

\section{LAVAGE-CENTRIFUGATION DE SPERMATOZOIDES NON SELEC- TIONNES}

Cette technique très simple consiste à diluer le sperme dans un milieu adéquat et de le centrifuger pendant 10 minutes à 300 ou $600 \mathrm{~g}$ suivant les auteurs. Sauf cas particuliers tels que les oligoasthénozoospermies très sévères [25], cette méthode est à proscrire car elle permet seulement d'éliminer le plasma séminal et expose les spermatozoïdes “ normaux " aux peroxydations membranaires générées par les leucocytes et les spermatozoïdes déficients [1, $2,3,4]$. De plus, la centrifugation par ellemême a des effets néfastes particulièrement nets à partir de $600 \mathrm{~g}[7,40,44,54]$.

\section{METHODES DE FILTRATION ET D'ADHERENCE}

La filtration sur laine de verre décrite en 1977 par Paulson et Polakoski [60] repose sur le principe de l'adhésion au verre des spermatozoïdes morts et des débris cellulaires. Cette technique a fait l'objet de quelques publications dans son emploi en AMP [76] et plus récemment Van der Bergh et coll. [75] l'ont utilisée avec succès dans la préparation du sperme avant injection intracytoplasmique de spermatozoïde (ICSI). Une étude récente [71] lui reconnaît des avantages supérieurs à la centrifugation sur gradient de Percoll, ce qui va à l'encontre des observations de Sherman et coll. [68]; ces auteurs avaient décrit des lésions de la membrane plasmique de la tête du spermatozoïde ainsi que des anomalies de l'acrosome probablement imputables à la filtration sur laine de verre d'où l'abandon de cette technique jusqu'à une date récente. La filtration sur billes de verre $[13,23,24]$ ne semble guère avoir emporté la conviction des différentes équipes l'ayant testée. 
La filtration sur colonne Sephadex («SpermPrep») est utilisée par quelques équipes qui lui trouvent une équivalence en termes d'efficacité par rapport à la centrifugation sur gradient de Percoll mais avec une plus grande rapidité d'exécution [18, 70, 80]. Une réserve doit cependant être émise pour l'usage en clinique en raison du rôle délétère observé sur la fécondation in vitro et le développement des embryons de souris incubés dans le milieu recueilli après rinçage de la colonne de filtration [67]; d'autre part, un effet négatif sur la mobilité des spermatozoïdes était observé. Même si ce milieu de rinçage de la colonne n'est pas utilisé pour la préparation du sperme, on ne peut exclure la persistance de facteurs délétères à l'état de traces dans le support de filtration.

\section{MIGRATION ASCENDANTE.}

La migration ascendante peut s'effectuer à partir du sperme ou à partir d'un culot de spermatozoïdes obtenus après un ou deux lavages centrifugation.

La migration ascendante à partir du sperme consiste à déposer le sperme sous le milieu de migration et à laisser migrer les spermatozoïdes pendant une heure à $37^{\circ} \mathrm{C}$. L'utilisation de plusieurs tubes pour augmenter l'interface sperme-milieu permet d'améliorer le rendement de la technique $[16,26]$. Cette méthode est supérieure à la migration à partir d'un culot de spermatozoïdes en terme de concentration en spermatozoïdes mobiles progressifs à morphologie normale en particulier pour le pourcentage d'acrosomes normaux [39]. Dans le cadre d'une FIV, il sera nécessaire de réaliser un lavage-centrifugation des spermatozoïdes recueillis avec le milieu de migration qui risquent d'entraîner avec eux un film de plasma séminal qui pourrait avoir un effet négatif sur la fécondation par la présence de facteurs décapacitants [42].

La migration des spermatozoïdes après lavage-centrifugation est utilisée depuis longtemps en FIV [48] et s'est largement répandue. Après la centrifugation, le surnageant est éliminé et $1 \mathrm{ml}$ de milieu approprié est déposé au-dessus du culot spermatique. La durée de la migration est de 30 minutes à une heure selon la qualité du sperme, la migration peut être faite à température ambiante ou mieux à $37^{\circ} \mathrm{C}$ dans une atmosphère contenant $5 \%$ de $\mathrm{C} 02$. La partie du milieu de migration la plus éloignée du culot est celle qui est récupérée de préférence à la partie la plus proche du culot que l'on peut être amené à utiliser si le nombre de spermatozoïdes récupérés dans la partie supérieure est trop faible. Russel et Rogers [66] ont proposé de remettre en suspension dans un faible volume de milieu $(0,2$ $0,3 \mathrm{ml}$ ) le culot obtenu après lavage et de déposer ce mélange au fond d'un tube contenant 2 $\mathrm{ml}$ de milieu. La remise en suspension des spermatozoïdes après centrifugation permettrait aux gamètes mobiles de s'extraire plus facilement du culot pour migrer, en particulier en cas d'asthénozoospermie, alors que les gamètes immobiles sédimentent; afin d'augmenter l'interface spermatozoïdes milieu de migration, le tube est incliné à 30-60 degrés et les conditions de temps et d'environnement sont les mêmes que pour la méthode de migration décrite précédemment. Quelle que soit la variante utilisée, la migration ascendante après lavage-centrifugation expose les spermatozoïdes aux dommages liés à la centrifugation et aux peroxydations membranaires $[2,3,7]$.

En outre, ces techniques ont un faible rendement, ce qui est un inconvénient en cas d'oligo et/ou asthénozoospermie ; Tea et coll. [73] ont décrit une méthode dite de migration sédimentation pour améliorer le rendement en cas de spermes déficients. Cependant, les techniques de centrifugation en gradient de densité lui sont préférées actuellement dans de telles situations.

\section{LES GRADIENTS DE DENSITE.}

Divers composés ont été utilisés pour la constitution des gradients de densité. A l'heure actuelle sont employés la silice colloïdale et le iodixanol. Le "Percoll" (Pharmacia-Sigma) est constitué de silice colloïdale recouverte de polyvinylpyrrolidone $[61,62]$ Son usage doit être strictement limité aux tests de migrationsurvie (TMS) à visée diagnostique car certains lots peuvent contenir des quantités non négligeables d'endotoxines. En vue d'AMP, le 
«PURESPERM» (NidaCon, Göteborg, Suède) ou «l'ISOLATE» (Irvine Scientific, Santa Ana, CA, USA), qui sont également des solutions de silice colloïdale recouverte de silane et agréés pour un usage humain, doivent être utilisés. Les résultats obtenus avec ces deux produits sont comparables à ceux du «Percoll» [14, 19].

Appliquée aux spermatozoïdes humains pour la première fois en 1981 par Gorus et Pipeleers [34], la préparation sur gradient de Percoll en vue d'AMP a connu un grand développement avant que son usage n'en soit restreint. Des simplifications $[9,29]$ ont été apportées à la méthode initiale (emploi d'un gradient discontinu à 6 densités) et plusieurs auteurs proposèrent des gradients simplifiés à 2 ou 3 phases de densités différentes [27, 53, 57,63]. Cette simplification apparaissait bénéfique dans les oligoasthénozoospermies sans rien faire perdre des avantages de la technique originelle du «Percoll» à 6 couches [52]:

- le plasma séminal et les cellules autres que les spermatozoïdes sont retenus dans les parties hautes du gradient (faible densité),

- les spermatozoïdes morts ou à mobilité déficiente sont retrouvés dans ces mêmes fractions de faible densité.

- dans les fractions de forte densité sont concentrés les spermatozoïdes les plus mobiles, avec de très bonnes caractéristiques morphologiques et présentant une bonne maturité nucléaire avec une fécondance supérieure aux spermatozoïdes sélectionnés par migration ascendante $[5,9,10,17,45$, $46,65,72]$. Très récemment [19], une étude a soulevé l'hypothèse de lésions membranaires liées au Percoll et un autre travail [71] a décrit des anomalies de l'acrosome et du flagelle plus fréquentes après Percoll qu'après filtration sur laine de verre. Ces publications vont à l'encontre des données généralement admises.

La technique de centrifugation sur gradient la plus employée actuellement consiste à utiliser un gradient à 2 densités ( 45 et $90 \%$ - pourcentages définis par rapport à la solution mère isoosmotique dite $100 \%$ ) et à centrifuger le sperme déposé au dessus du gradient, à $300 \mathrm{~g}$ pendant 20 minutes $[52,54]$. Le lavage-centri- fugation de la fraction de haute densité contenant les spermatozoïdes les plus mobiles est simple ou double suivant les auteurs et les vitesses et temps de centrifugation sont variables (de $150 \mathrm{~g}$ à $600 \mathrm{~g}$ et de 5 à 10 minutes); il semble préférable de ne pas aller au -delà de $400 \mathrm{~g}$ pour limiter les effets délétères de la centrifugation sans descendre en dessous de $300 \mathrm{~g}$ pour ne pas perdre en efficacité [51].

Il a été proposé diverses améliorations pour les oligoasthénozoospermies parmi lesquelles le recours à des gradients hyperosmotiques [15, 77] mais leur pratique ne s'est pas répandue.

\section{GRADIENTS DE IODIXANOL}

Le Percoll étant maintenant déconseillé pour un usage thérapeutique chez l'humain, le recours à des nouvelles techniques de préparation des spermatozoïdes s'est avéré indispensable. Hormis les nouvelles solutions de silice colloïdale commercialisées sous l'appellation «PureSperm» et «Isolate» qui remplacent le "Percoll» en AMP, de nouvelles molécules sont proposées. Des molécules iodées classiquement utilisées comme produits de contraste radiologique peuvent trouver leur place en AMP pour confectionner des gradients de densité. Le metrizamide, le iohexol (Nycodenz) et le iodixanol qui est un dimère de Nycodenz (Optiprep“), sont des molécules à forte densité candidates pour une utilisation en AMP [30,36].

Au cours de la centrifugation d'un gradient de densités, les spermatozoïdes migrent pour s'arrêter à l'endroit où le milieu qui les entoure est de la même densité que les spermatozoïdes: on dit que la centrifugation des spermatozoïdes est isopycnique. Dans le cas des gradients de silice colloïdale («Percoll»), la phase de plus forte densité est d'une densité $(1,10 \mathrm{~g} / \mathrm{ml})$ inférieure à celle des spermatozoïdes qui est en moyenne de $1,12 \mathrm{~g} / \mathrm{ml}$ [58], il y a donc culottage des cellules. Même les forces de centrifugation les plus élevées sont incapables d'entrainer les spermatozoïdes au-delà de leur point d'équilibre de densité avec le milieu qu'ils traversent. C'est ce principe de centrifugation qui a été repris pour la confection des gradients d'»Optiprep". 
Le sperme entier est mélangé avec la solution à $100 \%$ d'»Optiprep" prête à l'emploi (1vol./1,2 vol.). Cette suspension spermatique d'une densité de $1,32 \mathrm{~g} / \mathrm{ml}$ est déposée au fond d'un tube conique et recouverte par deux fractions de 2 ml chacune de solutions d'»Optiprep» à $51 \%$ (densité $1,17 \mathrm{~g} / \mathrm{ml}$ ) et à $26 \%$ (densité $1,09 \mathrm{~g} / \mathrm{ml}$ ). La centrifugation de 20 minutes à $300 \mathrm{x}$ g permet d'observer les spermatozoïdes sélectionnés sous la forme d'un anneau cellulaire à l'interface des deux phases $51 \%$ et $26 \%$. Les spermatozoïdes sélectionnés (densité $1,12 \mathrm{~g} / \mathrm{ml}$ ) ne peuvent pas pénétrer la phase à $26 \%$ de densité trop faible $(1,09 \mathrm{~g} / \mathrm{ml})$.

Une caractéristique originale de la solution d'»Optiprep» est qu'elle pénètre les cellules aux membranes altérées, ce qui par définition la différencie d'une solution colloïdale. Les cellules altérées, spermatozoïdes et leucocytes ne seront donc pas retrouvées dans la sélection mais resteront dans le fond du tube car pénétrées par la solution d'Optiprep à $100 \%$ de densité forte.

La densité d'un spermatozoïde est représentée par sa tête, le flagelle n'influant quasiment pas sur la densité de la cellule. Pendant la maturation testiculaire et épididymaire des spermatozoïdes, ils acquièrent leur mobilité et leur capacité fécondante en même temps qu'augmente leur densité nucléaire. Avec des gradients de densité comme le Percoll, on sélectionne des spermatozoïdes ayant peu d'anomalies céphaliques et ayant une bonne condensation nucléaire [74] ce qui est corrélé avec une bonne fécondance des spermatozoïdes in vitro [9, 15, 72, 74]. Quand il s'agit des spermatozoïdes sélectionnés sur gradient d'»Optiprep», les gamètes ayant la meilleure condensation nucléaire sont également sélectionnés mais l'intégrité de leur membrane est indispensable.

La technique de sélection par "Optiprep» donne un rendement non significativement plus faible que les gradients de solution colloïdale [19] mais montrera peut être une supériorité en terme de fécondance des spermatozoïdes aux membranes intactes sélectionnés. Cette technique mérite d'être évaluée plus précisément.
VIII. QUELLE TECHNIQUE UTILISER ?

\section{SPERME NORMAL}

Lorsque le sperme est normal selon les critères OMS [56], la migration ascendante peut être utilisée pour préparer les spermatozoïdes en vue d'une IIU ou d'une FIV classique ou avec micromanipulation (ICSI).

\section{ANOMALIES NON SPÉCIFIQUES DU SPERME}

En revanche, la présence d'une oligo et /ou asthénozoospermie impose le recours au gradient de densité pour obtenir un nombre suffisant de spermatozoïdes mobiles à inséminer in utéro (IIU) ou in vitro (FIV).

Dans les oligoasthénotératozoospermies très sévères, les gradients de densité peuvent être utiles mais le lavage simple peut suffire pour obtenir le nombre de spermatozoïdes nécessaire à la microinjection [25].

\section{Cas particuliers :}

\section{- Autoimmunisation antispermatozoïdes}

Lorsqu'il existe une auto-immunisation antispermatozoïdes avec anticorps anti-spermatozoïdes (ACAS) fixés sur les gamètes mâles et/ou présents dans le plasma séminal, divers moyens ont été proposés pour limiter leur effet sur la fécondation. Pour que les ACAS présents dans le plasma séminal ne viennent se fixer sur les spermatozoïdes après l'éjaculation pendant le temps de la liquéfaction, l'éjaculat sera recueilli dans 5 à $10 \mathrm{ml}$ de milieu contenant de l'albumine [55]. Immédiatement après le recueil, le sperme dilué dans le milieu sera centrifugé pour éliminer le plasma séminal. Cette technique permet de diminuer significativement le taux de spermatozoïdes porteurs d'ACAS. D'autres auteurs affirment que l'incubation du sperme avec du Test-Yolk buffer permet également d'éliminer une partie des ACAS mais ces résultats restent à confirmer pour valider la technique [37, 81]. Les spermes ainsi préparés sont ensuite centrifugés sur un gradient de densité.

\section{- Présence de leucocytes.}

Il est préférable d'avoir recours à la technique 
de centrifugation sur gradient afin d'éviter la compaction des spermatozoïdes avec les leucocytes dans le culot spermatique comme ce serait le cas pour la technique de migration ascendante. Des traitements in vitro à visée antioxydante ont été proposés [8, 35, 38, 59], mais leur emploi en pratique clinique n'a pas encore été validé.

\section{- Sperme hypervisqueux.}

Certains spermes se liquéfient mal et posent un problème pour la mise en route de leur préparation. La méthode la moins agressive consiste à diluer le sperme puis à l'aspirer et le refouler plusieurs fois à l'aide d'une pipette Pasteur. Le passage forcé à travers une aiguille montée sur une seringue ou l'utilisation d'enzymes sont à proscrire car non dénués d'innocuité pour les spermatozoïdes. En particulier, les enzymes pourraient avoir une action mal contrôlée sur des protéines membranaires spermatiques et leur origine le plus souvent animale pose un problème de sécurité microbiologique.

\section{-Ejaculation rétrograde.}

Le $\mathrm{pH}$ urinaire est habituellement acide est par conséquent délétère pour les spermatozoïdes $[21,50]$. De manière à alcaliniser les urines, il est demandé au patient de prendre une cuillère à café de bicarbonate de soude la veille et le matin du recueil du sperme. Deux heures avant le prélèvement de sperme, le patient doit boire un litre d'eau plate en évitant les eaux minérales et ne pas uriner jusqu'au prélèvement. Il est parfois possible de rétablir le caractère antérograde de l'éjaculation [21] en demandant au patient de pratiquer une masturbation debout vessie pleine. En cas d'échec il faudra alors traiter rapidement les urines recueillies immédiatement après masturbation. L'osmolarité de l'urine sera contrôlée car un choc hypoosmotique peut altérer gravement la mobilité des gamètes. Après lavage-centrifugation des urines, le culot est remis en suspension dans un milieu approprié et centrifugé sur un gradient de densité [41].

\section{- Spermes infectés.}

L'infection du sperme est une contre-indication à l'AMP. Cependant le sperme contient fré- quemment des germes commensaux voire des pathogènes mais en faible quantité qu'il conviendra d'éliminer. La centrifugation sur gradient de «Percoll» $[11,29,63]$ et la migration ascendante $[28,78]$ permettent le plus souvent d'éliminer les bactéries mais peuvent être prises en défaut pour $E$. Coli et les entérocoques [33]. Par contre, les gradients de «Percoll» laissent passer les levures de type Candida Albicans [49].

\section{- Spermes cryoconservés}

La préparation des spermes cryoconservés nécessite quelques particularités. En effet, avant congélation le sperme est mélangé avec un milieu cryoprotecteur ayant une osmolarité très forte. L'objectif de la préparation des spermatozoïdes sera de restaurer au mieux la mobilité des gamètes après décongélation et pour cela il sera nécessaire de débarrasser lentement les spermatozoïdes du milieu cryoprotecteur. Plusieurs techniques de préparation des spermatozoïdes congelés sont utilisées mais une seule a été très précisément évaluée par l'équipe de JK Critser [31] et permet de préserver au mieux la mobilité des gamètes.

La préparation des spermes cryoconservés commence par l'étape de décongélation rapide de la paillette en la sortant de l'azote liquide et en la gardant dans la main (proche de $37^{\circ} \mathrm{C}$ ) jusqu'à la liquéfaction de son contenu. La technique de décongélation en immergeant la paillette dans un bain marie est à proscrire sauf en cas de paillettes complètement scellées, dites de haute sécurité.

Avant congélation, le sperme a été dilué volume à volume avec du milieu cryoprotecteur très hyperosmolaire, il est donc nécessaire après décongélation et avant de sélectionner les spermatozoïdes de faire redescendre l'osmolarité dans des valeurs proches de celles du plasma, $300 \mathrm{mOsm}$. C'est cette étape qui conditionnera la bonne restauration de la mobilité spermatique [31].

Pour cela, le contenu d'une paillette de sperme est dilué très progressivement avec un même volume de milieu aux alentours de $300 \mathrm{mOsm}$. Pour une paillette d'un volume de $200 \mu 1,8$ fois $25 \mu 1$ de milieu seront additionnés au contenu de la paillette séquentiellement toutes les 
minutes. Ensuite, cette suspension spermatique est déposée sur un gradient de densité et traitée classiquement.

\section{ORIENTATION THERAPEUTIQUE EN AMP.}

Le choix de la technique d'AMP prend en compte les facteurs féminins d'infertilité. Par exemple, l'absence de trompes chez une femme est bien sûr une contre-indication à la réalisation d'IA. Le nombre de spermatozoïdes qu'il est possible d'obtenir à partir du sperme est également très important dans le choix de la technique d'AMP à proposer. C'est pourquoi, il semblerait totalement illogique de proposer un acte thérapeutique à un couple pour lequel une évaluation préalable du sperme dans le cadre d'un test de sélection-survie n'aurait pas été réalisé. D'autres part, les IA sont à proposer en priorité même si la qualité du sperme est médiocre mais que le Test de Migration-Survie (TMS) permet d'obtenir un million de spermatozoïdes sélectionnés. Dans ce cas, la réalisation de 6 IA est importante à respecter puisque nécessaire à donner toutes ses chances de grossesse au couple notamment en cas d'altérations non spécifiques des caractéristiques du sperme.

La décision de la meilleure thérapeutique à proposer au couple sera donc prise en fonction du nombre total de spermatozoïdes mobiles recueillis dans la préparation du sperme en tenant compte $\mathrm{du}$ pourcentage de formes typiques. En effet, en cas de tératozoospermie majeure la fécondance spermatique ne pourrait pas correctement s'exprimer et il ne serait pas logique de proposer des inséminations intra-utérines si à cette tératozoospermie s'ajoutait un nombre de spermatozoïdes inséminés très faible.

Le TMS réalisé avant une AMP comprend en plus de l'étape de sélection, une évaluation de la survie des spermatozoïdes à 2 h., 4 h. et 24 h. après préparation. Cette survie est estimée sur la mobilité des gamètes aux différents temps. L'absence de survie au temps $4 \mathrm{~h}$ compromet les chances de fécondation en FIV classique et orientera plutôt vers l'ICSI. La survie à 24 heures pose cependant des problèmes de standardisation. Il semble nécessaire d'ajuster la concentration spermatique et d'estimer la survie dans un milieu à la composition bien définie. Actuellement, ces éléments de standardisation n'existent pas.

En ce qui concerne le nombre de spermatozoïdes mobiles sélectionnés au TMS, les chiffres suivants sont donnés à titre d'ordre de grandeur car chaque dossier doit être apprécié au cas par cas.

Lorsque la préparation d'un éjaculat contient au moins 1 x $10^{6}$ spermatozoïdes mobiles progressifs (mobilité de type “ $a$ " et “ b" selon l'OMS) [56] les inséminations intra-utérines peuvent être proposées. Un nombre de spermatozoïdes inférieur doit faire recourir au cumul de deux éjaculats recueillis à une heure d'intervalle. L'objectif pour les IA est de ne pas descendre en dessous de 0,5 x 106 spermatozoïdes mobiles inséminés.

La FIV classique nécessite le recueil d'au moins $0,5 \times 10^{6}$ de spermatozoïdes mobiles et entre 0,2 et $0,5 \times 10^{6}$ spermatozoïdes il sera nécessaire de recourir à la technique de FIV en microgouttes qui permet de n'inséminer que 5 000 à 10000 spermatozoïdes par ovocyte.

Enfin, l'ICSI demeure la seule possibilité thérapeutique lorsque moins de $0,2 \times 106$ spermatozoïdes mobiles sont récupérés ou qu'une tératozoospermie majeure est observée.

\section{CONCLUSION}

La mise en œuvre d'une AMP nécessite la réalisation préalable d'un test de migration survie ainsi que le précise le texte du "Guide de Bonnes Pratiques de l'AMP" (J.O. du 28.02.99). Ce test permettra de déterminer la meilleure méthode de préparation des spermatozoïdes pour le patient concerné et conditionnera le choix d'une AMP. Bien sûr, ce choix ne reposant pas uniquement sur le résultat du test de migration-survie, d'autres investigations diagnostiques peuvent s'envisager.

Deux méthodes principales sont actuellement utilisées : la migration ascendante et la centrifugation sur gradient de silice colloïdale. Les biologistes doivent rester vigilants sur l'emploi de certains produits et il est recommandé d'uti- 
liser des milieux prêts à l'emploi et agréés pour un usage humain. De même, il faut éviter toute supplémentation des milieux commercialisés par des molécules dont l'effet n'a pas été préalablement validé par une expérimentation.

\section{ABSTRACT}

\author{
Selection Procedures of spermatozoa before \\ assisted reproductive \\ technologies
}

\section{C.MATHIEU, H.LUCAS}

There are many procedures to select spermatozoa but those procedures are often specific of each andrology centre. This review that is not an exhaustive review of the numerous methods published is rather devoided to focus on the main techniques, the sperm washing, the filtration methods ( "SpermPrep"), the swim-up, and the discontinuous density gradients with colloïdal solutions («Percoll», PureSperm" and «Isolate») including iodinated organic molecule as "OptiPrep". The first part presents a synthesis of literature in order to bring out standardized procedures, and a summary of the advantages and disadvantages of each step of these procedures.

Some specific anomalies of sperm need particular treatments. There is a consensus on the validated techniques to prepare semen samples in the presence of antisperm antibodies, leukocytes, bacterial contaminants, and also semen samples exhibiting hyperviscosity or in case of retrograde ejaculation. Cryopreserved sperm preparation is also described with references to experimental results in freezing-thawing human spermatozoa. The second part proposes guidelines for the therapeutic choice of the appropriate assisted reproductive technologies (i.e : intra uterine artificial insemination, in vitro fertilization or intracytoplasmic sperm injection) according to semen parameters, and to the outcome of sperm selection and survival.

Key-words : Human semen, sperm preparation, swim-up, density gradients, Percoll, filtration methods.

\section{REFERENCES}

1. AITKEN R.J., CLARKSON J.S. : Cellular basis of defective sperm function and its association with the genesis of reactive oxygen species by human spermatozoa. J. Reprod. Fertil. 1987, 81: 459-469.

2. AITKEN R.J., CLARKSON J.S. : Significance of reactive oxygen species and anti oxidants in defining the efficacy of sperm preparation techniques. J Androl., 1989, $9: 367-376$.

3. AITKEN R.J., CLARKSON J.S. FISHEL, S. : Generation of reactive oxygen species, lipid peroxidation and human sperm function. Biol. Reprod., 1989, 41 : 183-197.

4. AITKEN R.J., WEST K.M. : Analysis of the relationship between reactive oxygen species production and leucocyte infiltration in fractions of human semen separated on Percoll gradients. Int. J. Androl., 1990, $13: 433-451$.

5. AKERLOF E., FREDRICSSON B., GUSTAFSSON O., et al.. Comparison between a swim-up and a Percoll gradient technique for the separation of human spermatozoa. Int. J. Androl, 1987, 10 : 663-669.

6. ALTURA B.M., CARELLA A., ALTURA B.T.: Adverse effects of TRIS.HEPES and MOPS buffers on contractile response of arterial and venous smooth muscle induced by prostaglandins. Prostaglandins Med., 1980, 5 : 123-130.

7. ALVAREZ J.G., LASSO J.L., BLASCO L. et al. : Centrifugation of human spermatozoa induces sublethal damage ; separation of human spermatozoa from seminal plasma by a dextran swim-up procedure without centrifugation extends their motile lifetime. Hum. Reprod., 1993, 8 : 1087-1092.

8. BAKER H.W.G., BRINDLE J., IRVINE D.S., AITKEN R.J. : Protective effects of antioxidants on the impairment of sperm motility by activated polymorphonuclear leukocytes. Fertil. Steril., 1996, 65 : 411-419.

9. BERGER T., MARRS R.P., MOYER D.L. : Comparison of techniques for selection of motile spermatozoa. Fertil. Steril., 1985, $43: 268-273$.

10.BOLTON V.N., BRAUDE P.R. : Preparation of human spermatozoa for in vitro fertilization by isopycnic centrifugation on self generating density gradients. Arch. Androl., 1984, $13: 167-176$.

11.BOLTON V.N., WARREN R.E., BRAUDE P.R. : Removal of bacterial contaminants from semen for in vitro fertilization or artificial insemination by the use of buoyant density centrifugation. Fertil. Steril. 1986, $46: 1128-1132$.

12.BOWMAN C.M., BERGER E.M., BUTLER E.N., TOTH K.M., REPINE J.E. : HEPES may stimulate cultured endothelial cells to make growth-retarding oxygen metabolites. In Vitro Cell Dev. Biol. Steril. 1985, 21 Pt1 : 140-142.

13.CALAMERA J.C., QUIROS M.C., BRUGO S., 
NICHOLSON R.F. : Comparison between swim-up and glass bead column techniques for the separation of human spermatozoa. Andrologia, 1991, $23: 259$ 261.

14. CENTOLA G.M., HERKO R., ANDOLINA E., WEISENSEL S. : Comparison of sperm speration methods : effects on recovery, motility, motion, parameters and hyperactivation. Fertil. Steril., 1998, 70 : 1173-1175.

15. CHAN Y.N., ABUZEID M.I., MALCOMNSON J.H., SASY M.A. : Selection of human spermatozoa by a hyperosmotic two-layer Percoll gradient. Fertil. Steril., 1994, 61 : 1097-1102.

16. CHAN S.Y.W., CHAN Y.M., TUCKER M.J. : Comparison of characteristics of human spermatozoa selected by the multiple tube swim-up and simple discontinuous Percoll gradient centrifugation. Andrologia, 1991, $23: 213-218$.

17. CHAN S.Y.W., TUCKER M.J. : Differential sperm performance as judged by the zona free hamster egg penetration test relative to differing sperm penetration techniques. Hum. Reprod., 1992, 7 : 255-260.

18. CHECK J.H., ZAVOS P.M., KATSOFF D., KIEFER D. : Effects of Percoll Discontinuous Density Gradients vs Sperm prep II vs Sephadex G-50 gel infiltration on semen parameters. Arch. Androl., 1993, $31: 69-73$.

19. CLAASSENS O.E., MENKVELD R., HARRISO K.L. : Evaluation of three substitutes for Percoll in sperm isolation by density gradient centrifugation. Hum. Reprod., 1998, 13 : 3139-3143.

20. COWAN A.I., MARTIN R.L. : Ionic basis of the membrane potential response of rat dorsal vagal motoneurones to HEPES buffer. Brain Res., 1996, 717 : 69-75.

21. CRICH J.P., JEQUIER A.M. : Infertility in men with retrograde ejaculation : the action of urine on sperm motility and a simple method for achieving antegrade ejaculation. Fertil. Steril., 1978, 30 : 572-576.

22. DANIEL P.F. : Incorporation of labeled glucosamine into glycoproteins by organ cultures of hamster trachea: adverse effects of HEPES buffer. In Vitro., 1975, $11: 347-353$.

23. DAYA S., GWATKIN R.B.L. : Improvement in semen quality using glass bead column. Arch. Androl., 1987, $18: 241-244$.

24. DAYA S., GWATKIN R.B.L., BISSESSAR H. : Separation of motile human spermatozoa by means of a glass bead column. Gam. Res., 1987, $17: 375$ 380 .

25. DE VOS A., NAGY Z.P., VAN DE VELDE H., JORIS H., BOCKEN G., VAN STEIRTEGHEM A. : Percoll gradient centrifugation can be omitted in sperm preparation for intracytoplasmic sperm injection. Hum. Reprod., 1997, 12 : 1980-1984.

26. DODSON W.C., MOESSNER J., MILLER J. et al. : A randomized comparison of the methods of sperm preparation for intra-utérine insemination. Fertil. Steril., 1998, $70: 574-575$.

27. DRAVLAND J.E., MORTIMER D. : A simple discontinuous Percoll gradient for washing human spermatozoa. IRCS Med. Sci., 1985, 13 : 16-17.

28. FORMAN R., GUILLET-ROSSO F., FARI A., et al. Importance of semen preparation in avoidance of reduced in vitro fertilization results attributable to bacteria. Fertil. Steril., 1987, $47:$ 527-530.

29. FORSTER M.S., SMITH W.D., LEE W.I., et al. : Selection of human spermatozoa according to their relative motility and their interaction with zona free hamster eggs. Fertil. Steril., 1983, $40: 655-660$.

30. GELLERT-MORTIMER S.T., CLARKE G.N., BAKER H.W.G., et al. : Evaluation of Nycodenz R and Percoll density gradients for the selection of motile human spermatozoa. Fertil. Steril., 1988, 49 : 335-341.

31. GILMORE J.A., LIU J., GAO D.Y., CRITSER J.K. : Determination of optimal cryoprotectants and procedures for thei addition and removal from human spermatozoa. Hum. Reprod., 1997, 12 : 112-118.

32. GOMEZ E., AITKEN J. : Impact of in vitro fertilization culture media on peroxidative damage to spermatozoa. Fertil. Steril., 1996, $65: 880-882$.

33. GONZALES J., MATHIEU C., ZERAH S., VERDAGUER S. : Sperme, germes et PMA : une enquête des BLEFCO. Reprod. Hum. Horm., 1992, $3: 256-259$.

34. GORUS F.K., PIPELEERS D.G. : A rapid method for the fractionation of human spermatozoa according to their progressive motility. Fertil. Steril., 1981, 35 : 662-665.

35. GRIVEAU J.F., LE LANNOU D. : Effects of antioxidants on human sperm preparation techniques. Int. J. Androl., 1994, $17: 225-231$.

36. HATTORI $\mathrm{H}$, YAMAGUCHI $\mathrm{F}$, OHNO $\mathrm{H}$. AND NOMURA M. : Antigenecity study of iodixanol, a new non-ionioc contrast medium. J. of Toxicological Science 1995, 20 : suppl. 1, 117-123.

37. HENSLEIGH H.C., JAVKIN P.D., TAGATZ G.E., PRYOR J.L. : Evaluation for antisperm antibodies after storage of sperm in TEST-YOLK Buffer. Fertil. Steril., 1996, $66: 454-458$.

38. HUGHES C.M., LEWIS S.E.M., McKELVEY, MARTIN V.J., THOMPSON W. : The effects of antioxidant supplementation during Percoll preparation on human sperm DNA integrity. Hum. Reprod., 1998, $13: 1240-1247$.

39. JEULIN C., FENEUX D., SERRES C., et al. : Sperm factors related to failure of human in vitro fertilization. J. Reprod. Fertil., 1986, 76 : 735-744.

40. JONES R.C., HOLT W.V. : The effects of washing on the ultrastructure and cytochemistry of ram spermatozoa. J. Reprod. Fertil., 1974, 41: 159-167. 
41. JOUANNET P., SOUMAH A. : Ejaculation retrograde et fertilité in l'éjaculation et ses perturbations. J. Buvat, P. Jouannet Eds. SIMEP - Lyon., 1984, 62-68,

42. KANWAR K.C., YANAGIMACHI R., LOPATA A. : Effects of human seminal plasma on fertilizing capacity of human spermatozoa. Fertil. Steril., 1979, 31 : 321-327.

43. KIRSCH M., LOMONOSOVA E.E., KORTH H.G., SUSTMANN R., DE GROOT H. : Hydrogen peroxide formation by reaction of peroxynitrite with HEPES and related tertiary amines. Implications for a general mechanism. J. Biol. Chem., 1998, 273 : 1271612724 .

44. LEE W.I., GADDUM-ROSSE P., SMITH W.D., STEACHEVER M., BLANDAU R.J. : Laser light scattering study of the effect of washing on sperm motility. Fertil. Steril., 1982, $38: 62-67$.

45. LE LANNOU D., BLANCHARD Y. : Nuclear maturity and morphology of human spermatozoa selected by Percoll density gradient centrifugation or swim-up procedure. Reprod. Fertil., 1988, $84: 551-556$.

46. LESSLEY B.A., GARNER D.L. : Isolation of motile spermatozoa by density gradient centrifugation in Percoll. Gam. Res,, 1983, 7 : 49-61.

47. LEU P.L., REBEL G. : Effect of HEPES on the taurine uptake by cultured glial cells. J. Neurosci. Res., $1989,23: 78-86$.

48. MAHADEVAN M., BAKER G. : Assessement and preparation of semen for in vitro fertilization in Wood C., Trounson A. Eds. Clinical In vitro Fertilization. Springer Verlag. Berlin, 1984, p. 83-97.

49. MAHADEVAN M.M., BATRES F., MILLER M.M., MOUTOS D.M. : Yeast infection of sperm, oocytes and embryos after intra-vaginal culture for embryo transfer. Fertil. Steril., 1996, $66: 481-483$.

50. MAKLER A., DAVID R., BLUMENFELD Z., BETTER O.S. : Factors affecting sperm motility. VII. Sperm viability as affected by change of $\mathrm{pH}$ and osmolarity of semen and urine specimens. Fertil. Steril., 1981, 36 : 507-511.

51. MAKLER A., JACOBI P. : Effects of shaking and centrifugation on human sperm motility. Arch. Androl., 1981, $7: 2-6$.

52. MAKLER A., STOLLER J., MAKLER-SHIRAN E. : Dynamics aspects concerned with the mecanism of separating motile sperm from non motile sperm, leucocytes and debris with the use of high density Percoll gradients. Fertil. Steril., 1998, 70 : 961-966.

53. McCLURE R.D., NUNES L., TOM R. : Semen manipulation : improved sperm recovery and function with a two layer Percoll gradient. Fertil. Steril., $1989,51: 874-877$.

54. MORTIMER D. : Sperm recovery techniques to maximize fertilizing capacity. Reprod. Fertil. Dev., 1994, $6: 25-31$.
55. MORTIMER D. : Sperm preparation techniques and iatrogenic failures of in vitro fertilization. Hum. Reprod., 1991, $6: 173-176$.

56. O.M.S. : Manuel de laboratoire de l'OMS. Analyse du sperme humain et de l'interaction des spermatozoides avec le mucus cervical. Editions INSERM Paris, 1993.

57. ORD T., PATRIZIO P., MARELLO E., BALMACEDA J.P., ASCH R.H. : Mini-Percoll : a new method of semen preparation for IVF in severe male factor infertility. Hum. Reprod., 1990, 5 : 987-989.

58. OSHIO S. : Apparent densities of spermatozoa of various mammalian species. Gametes Research, 1988, $20: 1257-1259$.

59. PARINAUD J., LE LANNOU D., VIEITEZ G., GRIVEAU J.F., MILHET P., RICHOILLEY G. : Enhancement of motility by treating spermatozoa with an antioxidant solution («Sperm-Fit») following ejaculation. Hum. Reprod., 1997, 12 : 2434-2436.

60. PAULSON J.D., POLAKOSKI K.L. : A glass wool column procedure for removing extraneous material from the human ejaculate. Fertil. Steril., 1977, 28 : 178-181.

61. PERTOFT H., LAURENT T.C., LAAS T. : Density Gradients prepared from colloïdal silica particles coated by polyvynyl pyrolidone (Percoll). Anal. Biochem., $1978,88: 271-282$.

62. PICKERING S.J., FLEMING T.P., BRAUDE P.R., BOLTON V.N., GRESHAM G.A.G. : Are human spermatozoa separated on a Percoll density gradient safe for therapeutic use ? Fertil. Steril., 1989, 51 : 10241029 .

63. PUNJABI U., GERRIS J., VANBIJIEN J., DELBEKE L., GIELIS M., BUYTAERT P.H. : Comparison between different pre-treatment techniques for sperm recovery prior to intra uterine insemination, GIFT or IVF. Hum. Reprod., 1990, $5: 75-83$.

64. POOLE C.A., REILLY H.C., FLINT M.H. : The adverse effects of HEPES, TRIS, TES, and BES zwitterion buffers on the ultrastructure of cultured embryo epiphyseal chondrocytes. In Vitro, 1982, 18 : 755-765.

65. PRAKASH P., LEYKIN L., CHEN Z., et al. : Preparation by differential gradient centrifugation is better than swin-up in selecting sperm with normal morphology (strict criteria). Fertil. Steril,, 1998, 69 : $722-726$.

66. RUSSEL L.D., ROGER S.T. : Improvement in the quality and fertilization potential of a human sperm population using the rise technique. Androl., 1987, 8 : 25-33.

67. SCOTT L., SMITH S. : Mouse in vitro fertilization, embryo development and viability, and human sperm motility in substances used for human sperm preparation for assisted reproduction. Fertil. Steril., 1997, $67: 372-381$ 
68. SHERMAN J.K., PAULSON J.D., LIU K.C. : Effect of glass wool filtration on ultrastructure of human spermatozoa. Fertil. Steril., 1981, $36: 643-647$.

69. SIMPSON J.A., CHEESEMAN K.H., SMITH S.E., DEAN R.T. : Free-radical generation by copper ions and hydrogen peroxide. Stimulation by HEPES buffer. Biochem. J., 1988, $254:$ 519-523.

70. SOFIKITIS N., MIYAGAWA I., ZAVOS P.M. : Selection of single stranded deoxyribo nucleic acid spermatozoa via SpermPrep filtration column. Fertil. Steril. 1993, $59: 690-692$.

71. STERZIK K., DE SANTO M., UHLICH S., GAGSTEIGER F., STREHLER E. : Glass wool filtration leads to a higher percentage of spermatozoa with intact acrosomes : an ultrastructural analysis. Hum. Reprod., 1998, $13: 2506-2511$.

72. TANPHAICHITR N., MILLETTE C.F., AGULNICK A., FITZGERALD L.M. : Egg penetration ability and structural properties of human sperm prepared by Percoll gradient centrifugation. Gam. Res., 1988, 20 : 67-81.

73. TEA N.T., JONDET M., SCHOLLER R. : Procédé d'isolement des spermatozoïdes mobiles du sperme humain par la méthode de migration-sédimentation. Pathol. Biol., 1983, 31 : 688-690.

74. TOMLINSON M.J., BARRATT C.L.R., BOLTON A.E., et al. : Round cells and sperm fertilizing capacity : the presence of immature germ cells but not seminal leukocytes are associated with reduced sucess of in vitro fertilization. Fertili. Steril. 1992, $58: 1257-1259$.

75.VAN DER BERGH M., REVELARD P., BERTRAND E., BIRAMANE J., VANIN A.S., ENGLERT Y. : Glass wool column filtration, an advantageous way of preparing semen samples for intracytoplasmic sperm injection : an auto-controlled randomized study. Hum. Reprod., 1997, 12 : 509-513.

76. VAN DER VEN H.H., JEYENDRAN R.S., ALHASANI S., et al. : Glass wool column filtration of human semen : relation to swim-up procedure and outcome of IVF. Hum. Reprod., 1988, 3 : 85-88.

77. VELEZ DE LA CALLE J.F. - Human spermatozoa selection in improved discontinuous Percoll gradients. Fertil. Steril., 1991, 56 : 737-742.

78. WONG P.C., BALMACEDA J.P., BLANCO J.D., GIBBS R.S., ASCH R.H. : Sperm washing and swimup technique using antibiotics removes microbes from human semen. Fertil. Steril., 1986, 45 : 97-100.

79. YAMAMOTO D., SUZUKI N. : Blockage of chloride channels by HEPES buffer. Proc. R. Soc. Lond. B. Biol. Sci., 1987, 230 : 93-100.
80. ZAVOS P.M., CENTOLA G.M. : Améliorations séminales des spermatozoïdes utilisés pour la fécondation artificielle. Comparaison entre la méthode de nage ascendante et une nouvelle méthode de filtration du sperme sur colonne. Contracept. Fertil. Sex., 1990, $18: 943-948$.

81. ZAVOS P.M., CORREA J.R., ZARMAKOUPISZAVOS P.M. : Antisperm antibody treatment mode : levels of antisperm antibodies after incubation with TEST-YOLK Buffer and filtration using the SpermPrep II“ method. Fertil. Steril., 1998, 69 : 517521. 